\title{
Heart transplantation versus left ventricular assist devices as destination therapy or bridge to transplantation for 1-year mortality: a systematic review and meta-analysis
}

\author{
Christina A. Theochari ${ }^{1,2}$, George Michalopoulos ${ }^{1,2}$, Evangelos K. Oikonomou ${ }^{2}$, Stefanos Giannopoulos ${ }^{2}$, \\ Ilias P. Doulamis ${ }^{1,2}$, M. Alvarez Villela ${ }^{3}$, Damianos G. Kokkinidis ${ }^{2,4}$ \\ ${ }^{1}$ School of Medicine, University of Athens, Athens, Greece; ${ }^{2}$ Division of Internal Medicine, Society of Junior Doctors, Marousi, Greece; ${ }^{3}$ Division of \\ Cardiology, Montefiore Medical Center, ${ }^{4}$ Department of Medicine, Jacobi Medical Center, Albert Einstein College of Medicine, Bronx, NY, USA \\ Correspondence to: Damianos G. Kokkinidis, MD. 1955 Williambridge Rd, 6G, Bronx, NY, 10461, USA. Email: damiankokki@gmail.com.
}

\begin{abstract}
Background: The optimal treatment for advanced heart failure (HF) patients with regards to mortality remains unknown. Heart transplantation (HTx) and left ventricular assist devices (LVAD) used either as a bridge to transplant (BTT) or destination therapy (DT) have been compared in a number of studies, without definite conclusions with regards to mortality benefit. We sought to systematically review the pertinent literature and perform a meta-analysis of all the available studies presenting head-to-head comparisons between HTx and LVAD BTT or LVAD DT for late (>6 months) all-cause mortality.

Methods: We performed a systematic search of Medline and Cochrane Central databases in accordance with the Preferred Reporting Items for Systematic Reviews and Meta-Analyses (PRISMA) guidelines. We conducted a meta-analysis of late mortality comparing HTx vs. BTT LVAD and HTx vs. DT LVAD using a random effects model.

Results: Eight studies were included in our meta-analysis, reporting data on 7,957 patients in total. Although the available studies are of high quality [8 stars in Newcastle-Ottawa Scale (NOS) on average], there is paucity of mortality data. Specifically, seven studies compared HTx with BTT and five studies compared HTx with DT for 1-year mortality. Our pooled estimates showed that there was no difference in late mortality among these strategies.

Conclusions: Our meta-analysis highlights the small number and the heterogeneity of available studies referring to the optimal invasive management of advanced HF, and shows that there are no differences between HTx and LVAD for these patients with regards to late mortality.
\end{abstract}

Keywords: Heart failure (HF); heart transplantation (HTx); left ventricular assist devices (LVAD); bridge to transplantation (BTT)

Submitted Jun 26, 2017. Accepted for publication Sep 11, 2017.

doi: 10.21037/acs.2017.09.18

View this article at: http://dx.doi.org/10.21037/acs.2017.09.18

\section{Introduction}

Heart failure (HF) is a significant cause of morbidity and mortality that increases healthcare costs and leads to worsened quality of life, with a prevalence close to 23 million worldwide (1-4). Despite improvements in survival deriving from better medical therapies and prevention of sudden cardiac death, a percentage of patients with progressive HF continue to require heart transplantation
(HTx) or durable mechanical support [e.g., left ventricular assist devices (LVAD)] to prolong life (5). HTx remains the gold standard for end stage heart disease in adults. However, because of the paucity of donor hearts and the multiple common contraindications, this option is available only for a small fraction of patients (6,7). A ventricular assist device (VAD) is a type of durable mechanical circulatory support that partially replaces the function of the failing 
heart. Use of LVADs has grown steadily over the past decade since their initial approval for use as a bridge-totransplantation (BTT). Second and third generation LVADs have achieved improved durability through the use of wear free components, allowing for the upgrading of LVAD use to a destination therapy (DT) strategy in patients who are ineligible for HTx (8). LVADs provide patients with significant clinical improvement and prolong survival but are not free of complications (9). Data on 1-year mortality for patients managed with HTx vs. LVAD (BTT or DT) have been published by a number of observational, real world studies. Despite that, there is no clear evidence so far on the superiority of one of these strategies. With this systematic review and meta-analysis, we synthesize in a qualitative and quantitative manner all the available studies that present a head to head comparison between HTx and LVAD DT or HTx and LVAD BTT. Our aim is to reach informative conclusions regarding the potential superiority of one of the two strategies with regards to 1 -year survival.

\section{Methods}

This systematic review and meta-analysis was conducted according to Preferred Reporting Items for Systematic Reviews and Meta-Analyses (PRISMA) guidelines (10).

\section{Literature search and eligibility criteria}

Medline and Cochrane Central databases were reviewed by two reviewers independently (Christina A. Theochari and George Michalopoulos) for relevant articles published until February 28, 2017. The Medline algorithm used was the following: (VAD OR BiVAD OR LVAD OR ventricle-assist device OR "ventricle assist device" OR "ventricular assist device" OR "heart-assist device" OR "heart assist device" OR "heart-assist pump" OR "heart assist pump" OR "ventricular-assist device" OR "artificial ventricle" OR "mechanical circulatory support" ) AND ("heart transplantation" OR "heart grafting" OR "cardiac transplantation" OR "heart transplant" OR "cardiac transplant") AND ("heart failure" OR "myocardial failure" OR "cardiac failure" OR "heart decompensation").

The following inclusion criteria were used: (I) observational studies (real-world studies); (II) studies published in any language; (III) studies published up to February 28, 2017; (IV) studies presenting head to head comparisons between HTx and LVAD DT and/or HTx and LVAD BTT for HF patients; (V) studies that reported outcomes for one-year mortality. A study was considered eligible for the meta-analysis if fulfilled all the inclusion criteria. When there was a disagreement between the two reviewers (Christina A. Theochari and George Michalopoulos), a third reviewer (Evangelos Oikonomou) was involved in order to reach consensus. References from all the included studies and other relevant review papers were also manually reviewed in order to identify potentially additional eligible studies.

\section{Data extraction and quality assessment}

Data extraction from the eligible studies was performed by two investigators (Christina A. Theochari and George Michalopoulos) independently. Any disagreements were resolved by consensus. A standardized extraction form in an Excel spreadsheet was used. Data for the following variables were extracted: first author; year of publication; country and center; setting; study design and methodology; patient demographic characteristics (gender, age, race); HF etiology; LVAD strategy (BTT or DT); index hospitalization length; follow-up length after the primary intervention; readmission rate per year of support, stroke, infections, cost and mortality. The primary outcome of our meta-analysis was late mortality ( $>6$ months). The Newcastle-Ottawa Scale (NOS) was used to assess the quality of the observational studies included in our metaanalysis. The NOS has two different instruments for assessing case-control and cohort studies. Each instrument includes measures of quality in three domains: selection, comparability, and exposure. A study can receive up to one point for each of four areas measured within the selection domain and for each of three areas measured within the exposure domain. A maximum of two points can be assigned within the comparability domain. The highest possible score is nine. High-quality studies were considered to have a score of seven or greater. Study quality was assessed independently by two investigators (Christina A. Theochari and George Michalopoulos).

\section{Statistical analysis}

In order to calculate the risk estimates within each study for patients treated with HTx, LVAD DT or LVAD BTT, we extracted the absolute number of patients who had an event in each arm. For studies that reported only relative and not absolute frequencies, we used the total number of eligible patients in each arm to calculate the absolute frequencies of 


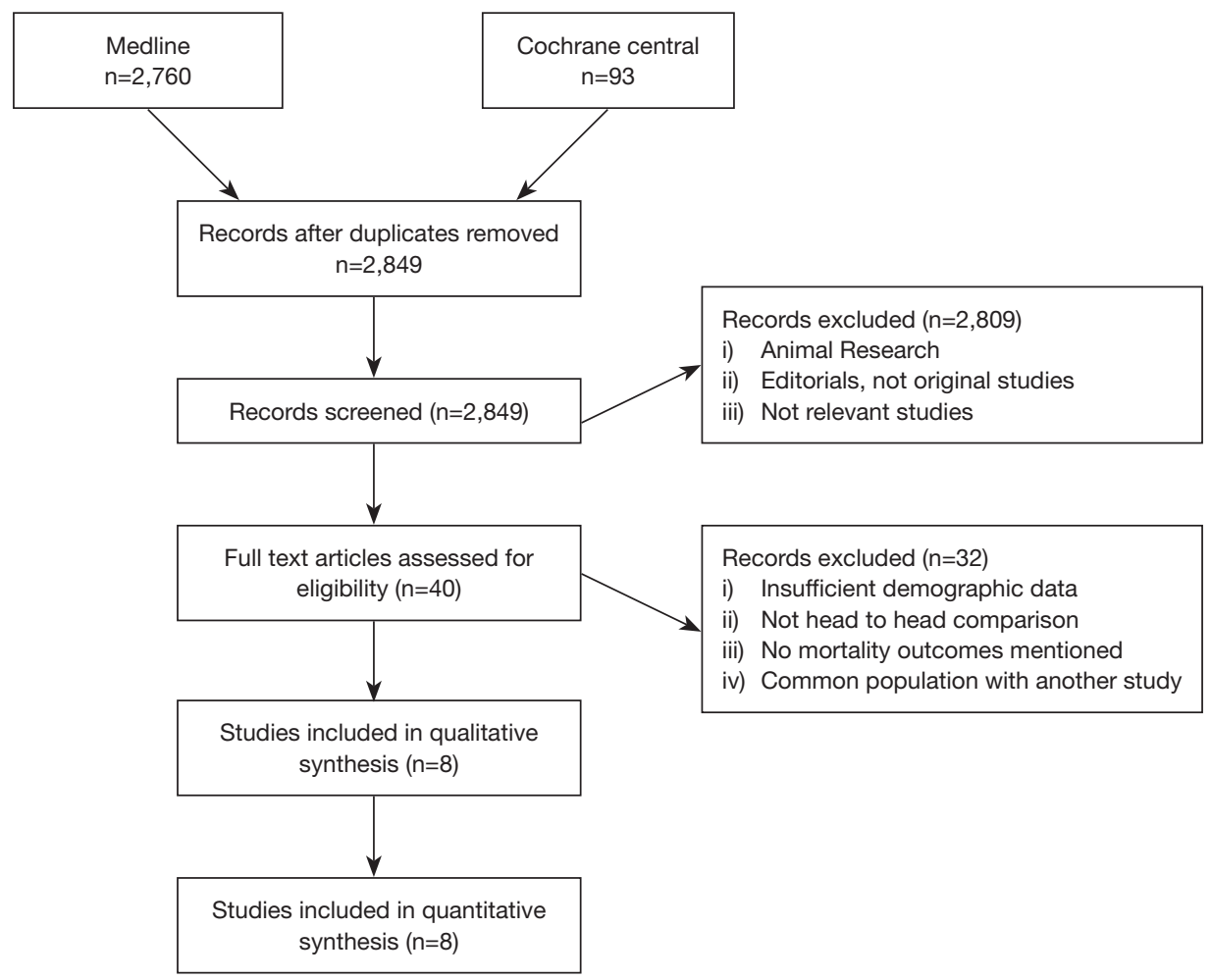

Figure 1 PRISMA flow diagram of the included studies. PRISMA, Preferred Reporting Items for Systematic Reviews and Meta-Analyses.

the events. Categorical variables are presented as absolute and relative frequencies and continuous variables as mean values $\pm \mathrm{SD}$. The estimated incidence rates were expressed as percentage and $95 \%$ confidence intervals (CI). A random effects model was used for our meta-analysis. Heterogeneity among studies was assessed with the $\mathrm{I}^{2}$ statistic. Values $<25 \%$ indicated non-significant heterogeneity, $25 \%$ to $50 \%$ indicated moderate, and $>50 \%$ indicated severe heterogeneity. Forest plots were used to present the overall effects. Both of our analyses included less than 10 studies (five studies for HTx vs. LVAD DT, and seven studies for HTx vs. LVAD BTT) while by definition the included studies were observational and not randomized controlled trials. We used funnel plots in an attempt to locate possible publication bias, despite the small number of the available studies for each outcome (Figure S1A,B) $(10,11)$. The results were regarded as statistically significant at a two-sided $\mathrm{P}<0.05$. We used STATA 14.1 (StataCorp, College Station, Texas, USA) as statistical software.

\section{Results}

Our searches yielded 2,760 studies from Medline and
93 from Cochrane Central. After removal of duplicates, we assess for eligibility 2,849 studies. We excluded 2,809 studies based on their title and abstract and 40 articles were retrieved for full-text review, before resulting in the eight eligible studies (11-18). The detailed flow diagram is presented in Figure 1. All studies were deemed to be of high quality on the basis of the NOS score; two were assessed with 9 stars, three with 8 stars and three with 7 stars. Table 1 summarizes the baseline characteristics of the included studies. Four were conducted in the USA and four in Europe. The eight included studies involved 7,957 patients in total; $74.2 \%$ were males. HTx was the treatment for 5,206, while 92 underwent LVAD as a DT and 2,737 LVAD as a BTT. The mean age for the patients ranged from 46 to 67.2 years in the individual studies. The follow-up period ranged from 12 to 50 months for the HTx group and from 9.4 to 30 months for the BTT LVAD group. Four studies reported data on left ventricular ejection fraction (LVEF). The mean LVEF ranged from $14 \%$ to $24 \%$ in the HTx group, from $15.6 \%$ to $20.4 \%$ for the BTT LVAD group and from $13 \%$ to $20 \%$ for the DT LVAD group. More detailed data from the individual studies are presented in Tables 2 and 3. Seven studies presented a head to head comparison 


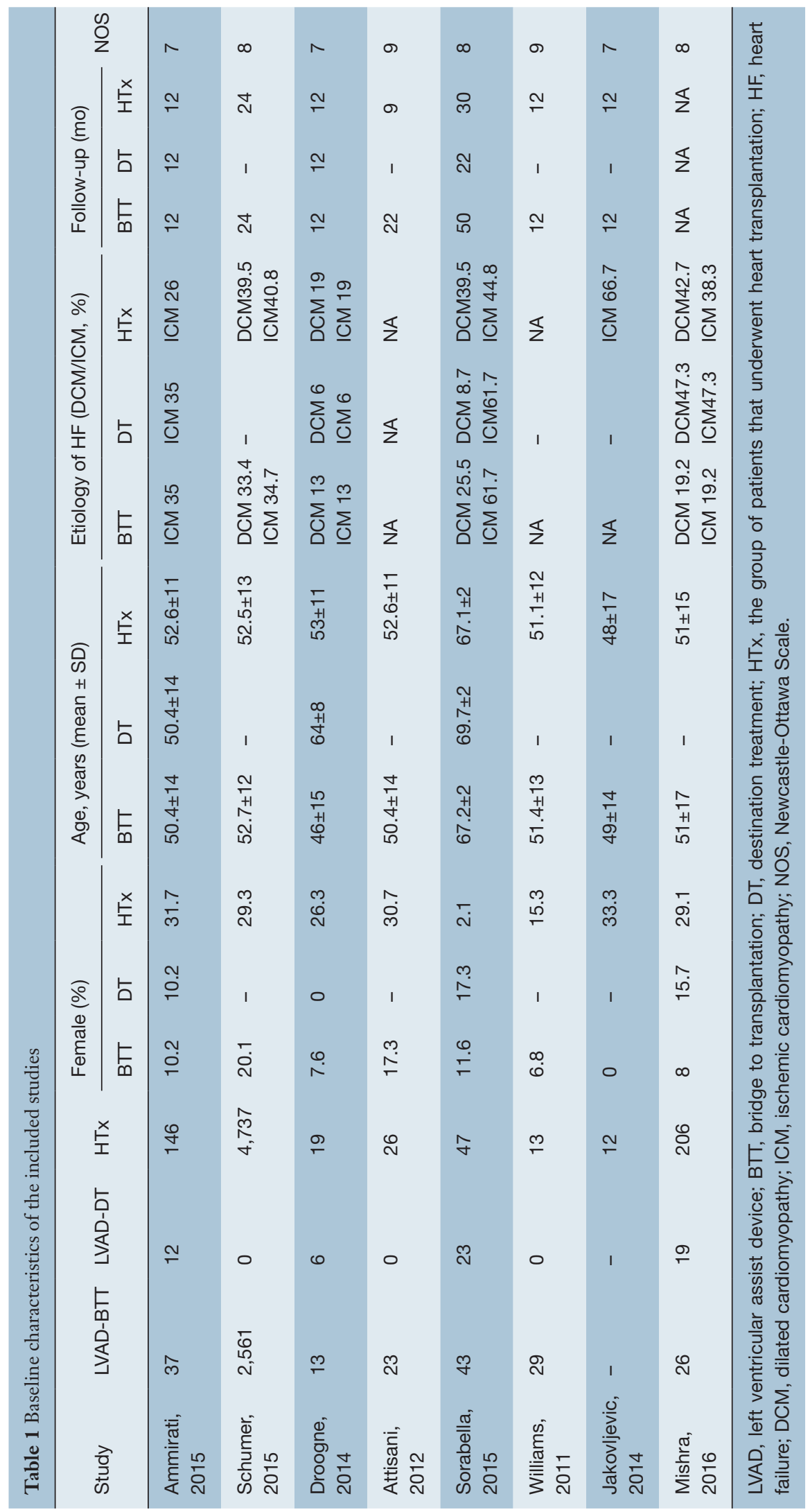




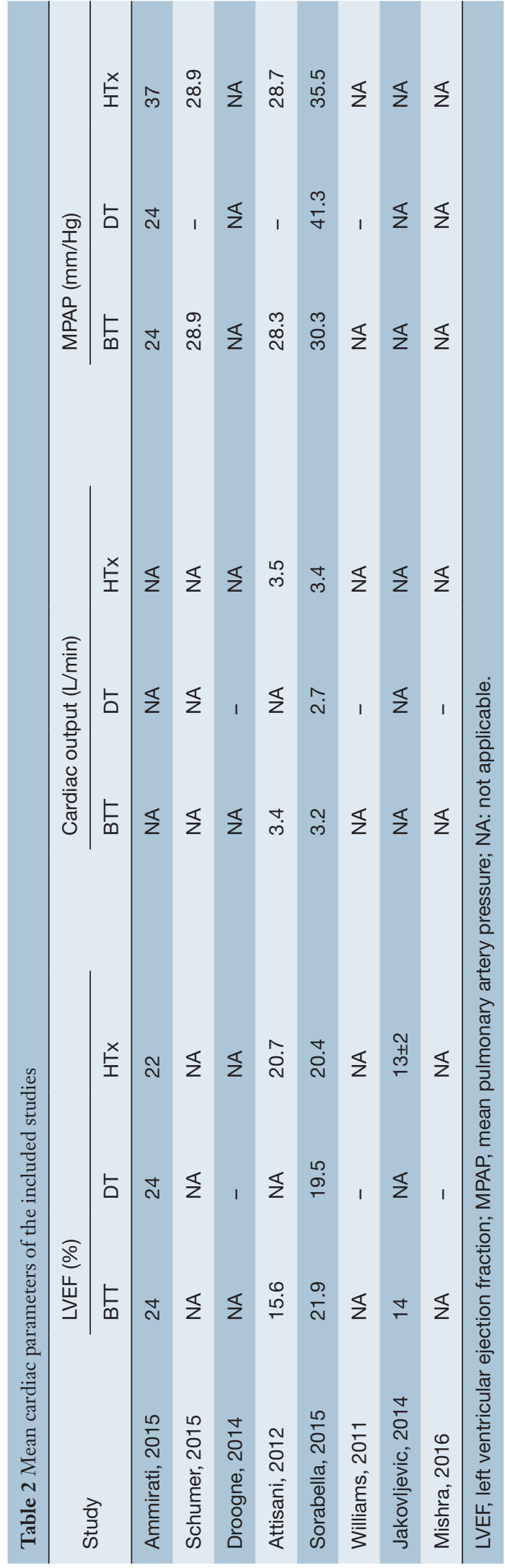

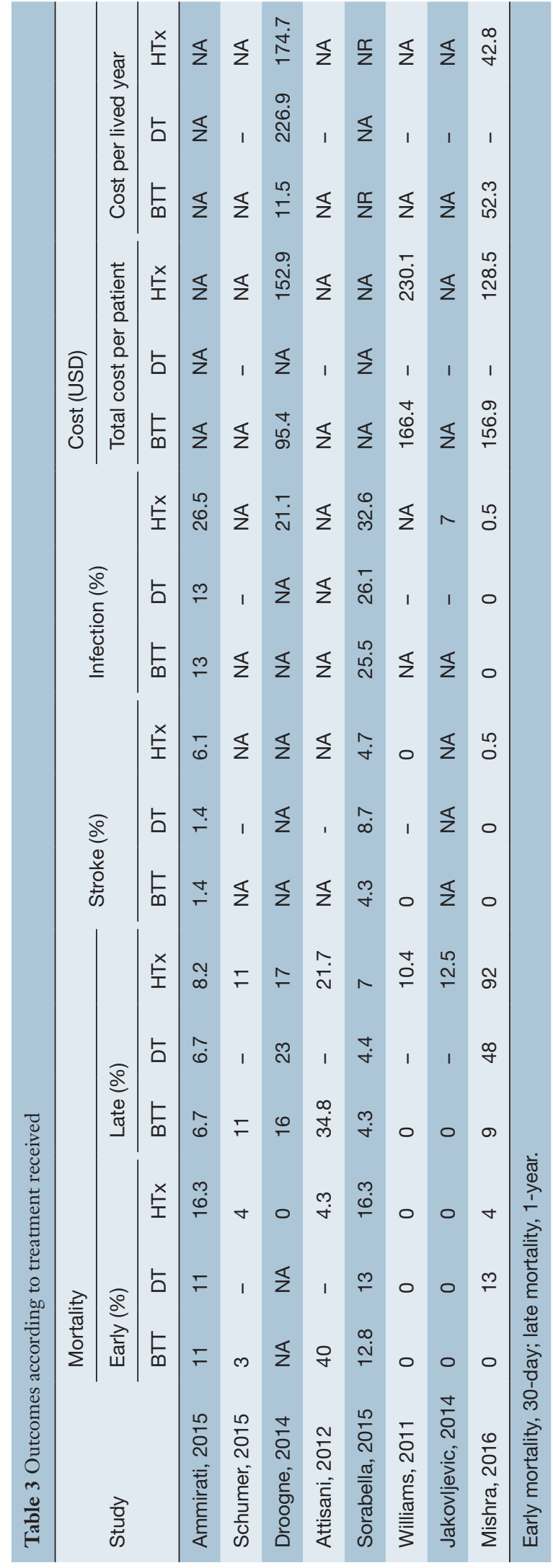




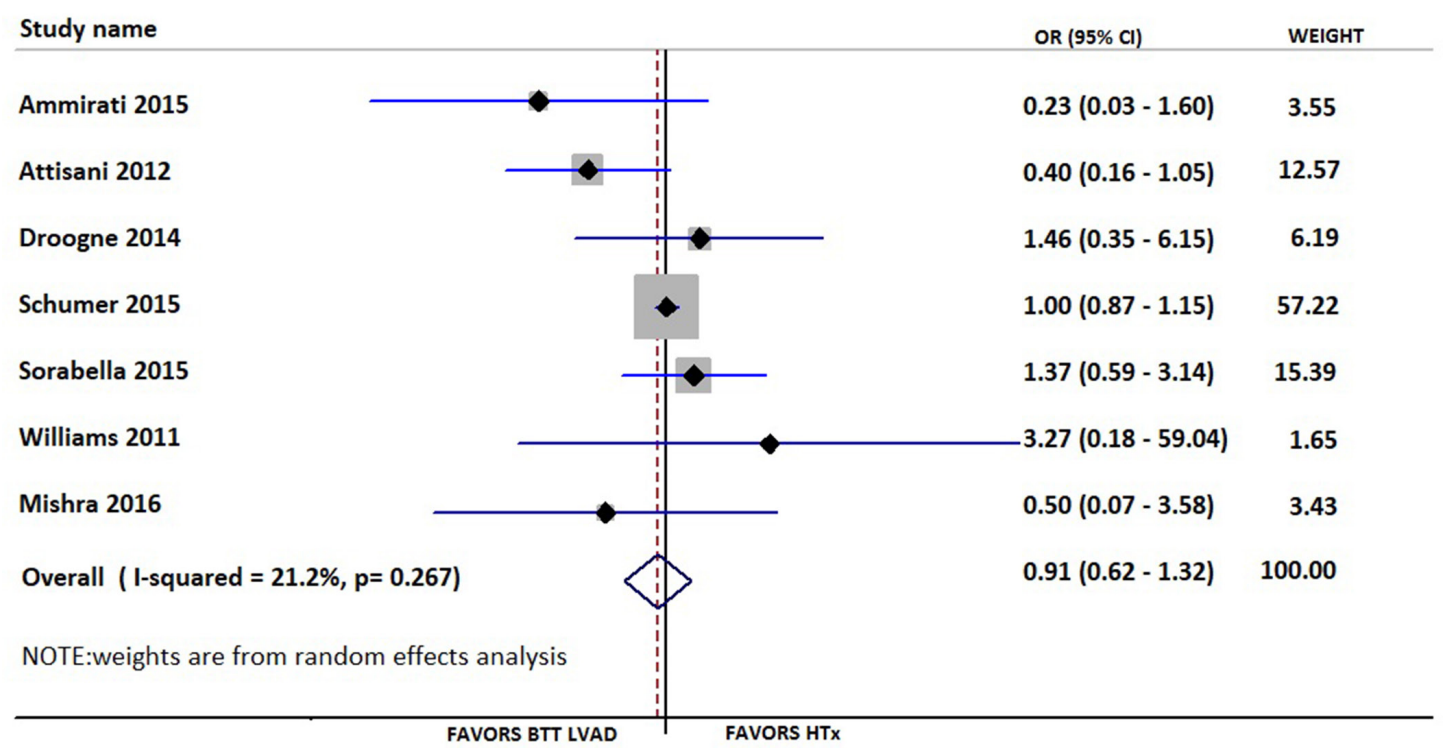

Figure 2 Forest plot for 1-year mortality between heart transplantation and LVAD BTT. There was no difference in 1-year mortality rates between BTT LVAD and HTx among seven studies (pooled OR: 0.91; 95\% CI: $0.62-1.32$; $\mathrm{I}^{2}=21.2 \%$ ). BTT, bridge to transplantation; LVAD, left ventricular assist devices; HTx, heart transplantation.

between HTx and LVAD BTT for 1-year mortality. Our meta-analysis showed that there was no significant difference in 1-year mortality rates between these two groups (pooled OR: 0.91; 95\% CI: 0.62-1.32) (Figure 2), without significant heterogeneity $\left(\mathrm{I}^{2}=21.2\right)$. Comparison data between HTx and LVAD DT, were reported by five studies. Our meta-analysis showed absence of significant differences in 1-year mortality rates also between these two groups (pooled OR: 1.49; 95\% CI: 0.48-4.66) (Figure 3), although the heterogeneity was significant here $\left(\mathrm{I}^{2}=82.8\right)$. Stroke and infection rates were reported by four studies. Stroke rates ranged from $0 \%$ to $4.3 \%$ in the HTx group, from $0 \%$ to $6.1 \%$ in the LVAD BTT group and from $4.3 \%$ to $6.1 \%$ in the LVAD DT group. Infection rates ranged from $0.5 \%$ to $25.5 \%$ in the HTx group, from $21.1 \%$ to $26.5 \%$ in the LVAD DT group and from $0 \%$ to $32.6 \%$ in the LVAD BTT patients. The total cost per patient was reported by three studies for the HTx group (range: 128,474 to 230,009 USD), by three studies for the LVAD BTT group (range: 95,428 to 166,415 USD) and by one study for the LVAD DT group (range: 152,931 to 174,165 USD).

\section{Discussion}

To the best of our knowledge, this is the first systematic review and meta-analysis on this topic. We included only studies presenting head to head comparisons between HTx and LVAD. We used the available data to perform two different and clinically meaningful comparisons: HTx vs. LVAD DT and HTx vs. LVAD BTT. Our findings show that there was no difference in 1-year mortality rates between LVAD BTT and HTx (pooled OR: 0.91; 95\% CI: $0.62-1.32 ; \mathrm{I}^{2}=21.2 \%$ ) or between LVAD DT and HTx (pooled OR: 1.49; 95\% OR: 0.48-4.66; $\mathrm{I}^{2}=82.8 \%$ ).

Previous published observational studies agreed with our results and indicated that there is not a statistically significant difference in 1-year mortality between HTx and BTT LVAD patients not only for 1-year mortality but also for early (30-day) or 2- and 5-year mortality. There is only one observational study (Attisani et al.) that supports a HTx superiority for in-hospital mortality (14). This last study compared patients on the waiting list for HTx with urgent conditions and patients managed with LVAD as a BTT. In-hospital mortality was found to be significantly higher for HTx waiting list patients compared to BTT LVAD patients ( $42.3 \%$ vs. $4.3 \%, \mathrm{P}=0.002$ ). Although almost all of the included studies, similarly to our overall results, did not find statistically significant differences in 1-year mortality between DT LVAD and HTx patients, Mishra et al. found that DT LVAD patients had inferior short term survival comparing to HTx (17). However, this study was limited by 


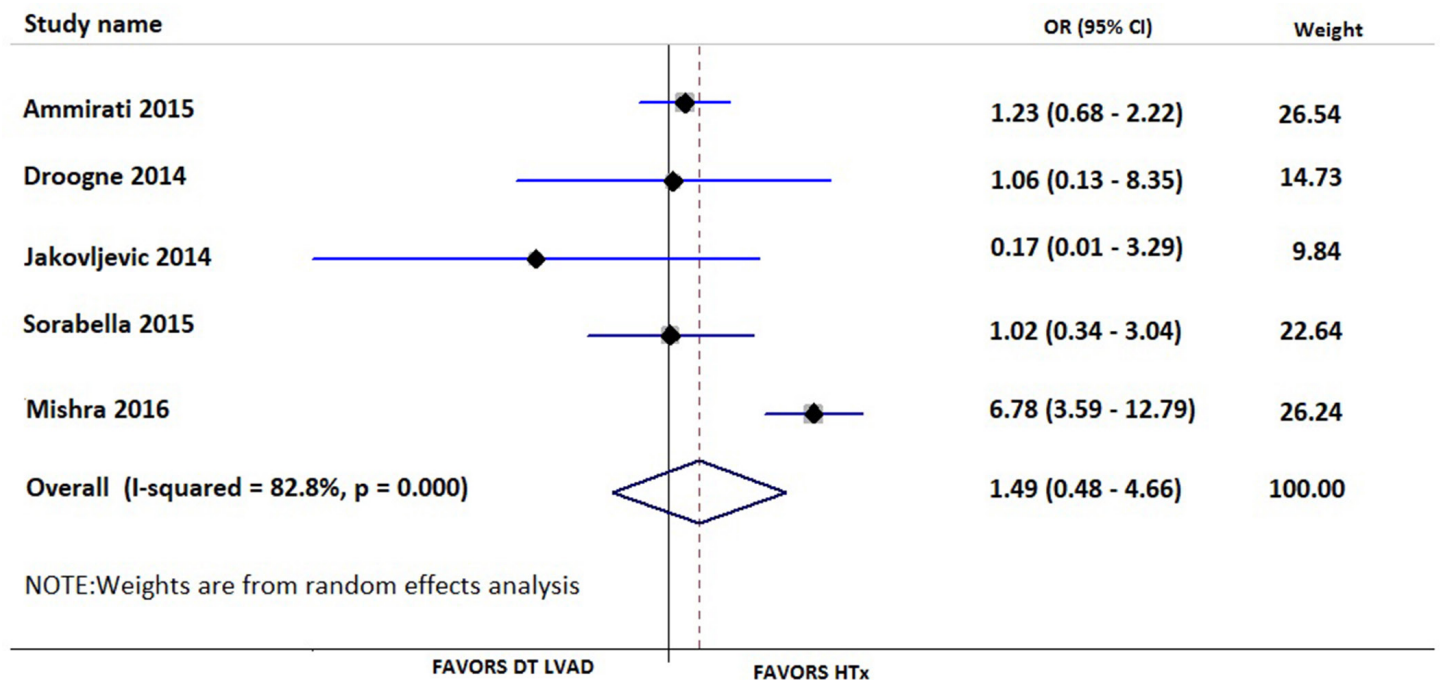

Figure 3 Forest plot for 1-year mortality between heart transplantation and LVAD DT. There was no difference in 1-year mortality rates between DT LVAD and HTx among five studies (pooled OR: 1.49; 95\% CI: 0.48-4.66; I² =82.8\%). DT, destination therapy.

its small size.

Although complications with LVAD therapy are not uncommon, most of them are manageable and current outcomes clearly support the use of LVAD in advanced HF $(19,20)$. On the other hand, HTx remains a promising treatment option for many patients but suitable donor availability remains extremely limited (21). There are important distinctions to be made between candidates for each treatment. For instance, pulmonary hypertension is a significant contraindication for transplantation but not for LVAD therapy (22). In contrast, patients with severe right ventricular failure are less optimal candidates for LVAD, but may experience good outcomes with transplantation (23-26). With regards to renal dysfunction, current data show that LVAD implantation can lead to an improvement (27). On the other hand, LVAD is not a panacea and there are multiple subgroups of patients who have contraindications for LVAD implantation, including those prone to infection, elderly patients and patients with untreated aortic regurgitation (28-31). Furthermore, LVAD implantation may be associated with increased tendency to ventricular tachyarrhythmia (32). Whether a distinction between BTT and DT patients is clinically meaningful, remains a questionable issue (33). There is a possibility that patients awaiting transplantation on LVAD support may develop contraindications to transplant, or never receive a suitable organ given the paucity of donors (24).

\section{Limitations}

This was a meta-analysis of real-world data and should thus be interpreted within the context of the known limitations of the observational research. Retrospective studies are associated with bias and endpoints for LVAD and HTx may not be directly comparable. In the absence of additional data, we included a small number of studies and patients with heterogeneous populations at baseline. Without knowing specific patient level characteristics of the included studies, we were unable to adjust for comorbidities and other baseline characteristics. Furthermore, the LVAD type was not the same in all the individual studies. In some studies, patients who were initially managed as BTT were converted to DT LVAD, either due to the paucity of available heart transplant, or other inhibitory conditions. This overlap limited our ability to clearly differentiate these two groups of patients. Last but not least, our funnel plots are not able to totally exclude or confirm publications bias, since they are limited by the small number of studies. Publication bias can be suspected, considering that research teams were less likely to publish insignificant or negative findings for the one or the other approach.

\section{Conclusions}

Despite the limitations, this systematic review and metaanalysis qualitatively and quantitatively synthesized the 
published evidence comparing the 1-year survival between HTx and LVAD either as bridge to transplantation or destination therapy. Both LVAD strategies seem to be noninferior to HTx. Unique limitations to each treatment strategy are likely to continue to dictate patient eligibility for each therapy in the absence of clear survival benefit differences. We anticipate that with the addition of more real-world data in the future, safer conclusions could be reached on this important clinical topic.

\section{Acknowledgements}

None.

\section{Footnote}

Conflicts of Interest: The authors have no conflicts of interest to declare.

\section{References}

1. Chen J, Normand SL, Wang Y, et al. National and regional trends in heart failure hospitalization and mortality rates for Medicare beneficiaries, 1998-2008. JAMA 2011;306:1669-78.

2. McMurray JJ, Adamopoulos S, Anker SD, et al. ESC Guidelines for the diagnosis and treatment of acute and chronic heart failure 2012: The Task Force for the Diagnosis and Treatment of Acute and Chronic Heart Failure 2012 of the European Society of Cardiology. Developed in collaboration with the Heart Failure Association (HFA) of the ESC. Eur Heart J 2012;33:1787-847.

3. Roger VL. Epidemiology of heart failure. Circ Res 2013;113:646-59.

4. Myserlis PG, Malli A, Kalaitzoglou DK, et al. Atrial fibrillation and cognitive function in patients with heart failure: a systematic review and meta-analysis. Heart Fail Rev 2017;22:1-11.

5. New York Heart Association. Criteria Committee. Nomenclature and criteria for diagnosis of diseases of the heart and great vessels. 8th ed. Boston: Little Brown and Company, 1979.

6. Hunt SA, Abraham WT, Chin MH, et al. 2009 focused update incorporated into the ACC/AHA 2005 Guidelines for the Diagnosis and Management of Heart Failure in Adults: a report of the American College of Cardiology Foundation/American Heart Association Task Force on Practice Guidelines: developed in collaboration with the
International Society for Heart and Lung Transplantation. Circulation 2009;119:e391-479.

7. Zaroff JG, Rosengard BR, Armstrong WF, et al. Consensus conference report. Circulation 2002;106:836-41.

8. Peura JL, Colvin-Adams M, Francis GS, et al. Recommendations for the use of mechanical circulatory support: device strategies and patient selection. Circulation 2012;126:2648-67.

9. Prinzing A, Herold U, Berkefeld A, et al. Left ventricular assist devices - current state and perspectives. J Thorac Dis 2016;8:E660-6.

10. Moher D, Liberati A, Tetzlaff J, et al. Preferred reporting items for systematic reviews and meta-analyses: the PRISMA statement. PLoS Med 2009;6:e1000097.

11. Ammirati E, Oliva FG, Colombo T, et al. Mid-term survival after continuous-flow left ventricular assist device versus heart transplantation. Heart Vessels 2016;31:722-33.

12. Schumer EM, Ising MS, Trivedi JR, et al. Early outcomes with marginal donor hearts compared with left ventricular assist device support in patients with advanced heart failure. Ann Thorac Surg 2015;100:522-7.

13. Droogne W, Jacobs S, Van den Bossche K, et al. Cost of 1-year left ventricular assist device destination therapy in chronic heart failure: a comparison with heart transplantation. Acta Clinica Belgica 2014;69:165-70.

14. Attisani M, Centofanti P, Torre ML, et al. Advanced heart failure in critical patients (INTERMACS 1 and 2 levels): ventricular assist devices or emergency transplantation? Interact Cardiovasc Thorac Surg 2012;15:678-84.

15. Jakovljevic DG, McDiarmid A, Hallsworth K, et al. Effect of left ventricular assist device implantation and heart transplantation on habitual physical activity and quality of life. Am J Cardiol 2014;114:88-93.

16. Williams ML, Trivedi JR, McCants KC, et al. Heart transplant vs left ventricular assist device in heart transplant-eligible patients. Ann Thorac Surg 2011;91:1330-3; discussion 1333-4.

17. Mishra V, Fiane AE, Winsnes BA, et al. Cardiac replacement therapies: outcomes and costs for heart transplantation versus circulatory assist. Scand Cardiovasc J 2017;51:1-7.

18. Sorabella RA, Yerebakan H, Walters R, et al. Comparison of outcomes after heart replacement therapy in patients over 65 years old. Ann Thorac Surg 2015;99:582-8.

19. Reiss N, Schmidt T, Müller-von Aschwege F, et al. Telemonitoring and Medical Care of Heart Failure Patients Supported by Left Ventricular Assist Devices The Medolution Project. Stud Health Technol Inform 
2017;236:267-74.

20. Lanfear DE, Levy WC, Stehlik J, et al. Accuracy of Seattle Heart Failure Model and HeartMate II Risk Score in NonInotrope-Dependent Advanced Heart Failure Patients: Insights From the ROADMAP Study (Risk Assessment and Comparative Effectiveness of Left Ventricular Assist Device and Medical Management in Ambulatory Heart Failure Patients). Circ Heart Fail 2017;10.

21. Blanche C, Kamlot A, Blanche DA, et al. Heart transplantation with donors fifty years of age and older. J Thorac Cardiovasc Surg 2002;123:810-5.

22. Murali S, Uretsky BF, Reddy PS, et al. Reversibility of pulmonary hypertension in congestive heart failure patients evaluated for cardiac transplantation: comparative effects of various pharmacologic agents. Am Heart J 1991;122:1375-81.

23. Cordtz J, Nilsson JC, Hansen PB, et al. Right ventricular failure after implantation of a continuous-flow left ventricular assist device: early haemodynamic predictors. Eur J Cardiothorac Surg 2014;45:847-53.

24. Drakos SG, Janicki L, Horne BD, et al. Risk factors predictive of right ventricular failure after left ventricular assist device implantation. Am J Cardiol 2010;105:1030-5.

25. Kormos RL, Teuteberg JJ, Pagani FD, et al. Right ventricular failure in patients with the HeartMate II continuous-flow left ventricular assist device: incidence, risk factors, and effect on outcomes. J Thorac Cardiovasc Surg 2010;139:1316-24.

26. Atluri P, Goldstone AB, Fairman AS, et al. Predicting

Cite this article as: Theochari CA, Michalopoulos G, Oikonomou EK, Giannopoulos S, Doulamis IP, Villela MA, Kokkinidis DG. Heart transplantation versus left ventricular assist devices as destination therapy or bridge to transplantation for 1-year mortality: a systematic review and meta-analysis. Ann Cardiothorac Surg 2018;7(1):3-11. doi: 10.21037/ acs.2017.09.18 right ventricular failure in the modern, continuous flow left ventricular assist device era. Ann Thorac Surg 2013;96:857-63; discussion 863-4.

27. Hasin T, Topilsky Y, Schirger JA, et al. Changes in renal function after implantation of continuous-flow left ventricular assist devices. J Am Coll Cardiol 2012;59:26-36.

28. Kirklin JK, Naftel DC, Pagani FD, et al. Seventh INTERMACS annual report: 15,000 patients and counting. J Heart Lung Transplant 2015;34:1495-504.

29. Cotts WG, McGee EC, Myers SL, et al. Predictors of hospital length of stay after implantation of a left ventricular assist device: An analysis of the INTERMACS registry. J Heart Lung Transplant 2014;33:682-8.

30. Holley CT, Fitzpatrick M, Roy SS, et al. Aortic insufficiency in continuous-flow left ventricular assist device support patients is common but does not impact long-term mortality. J Heart Lung Transplant 2017;36:91-6.

31. Pal JD, Klodell CT, John R, et al. Low operative mortality with implantation of a continuous-flow left ventricular assist device and impact of concurrent cardiac procedures. Circulation 2009;120:S215-9.

32. Sims DB, Uriel N, González-Costello J, et al. Human immunodeficiency virus infection and left ventricular assist devices: a case series. J Heart Lung Transplant 2011;30:1060-4.

33. Nakagawa S, Yuzefpolskaya M, Colombo PC, et al. Palliative Care Interventions before Left Ventricular Assist Device Implantation in Both Bridge to Transplant and Destination Therapy. J Palliat Med 2017;20:977-83. 

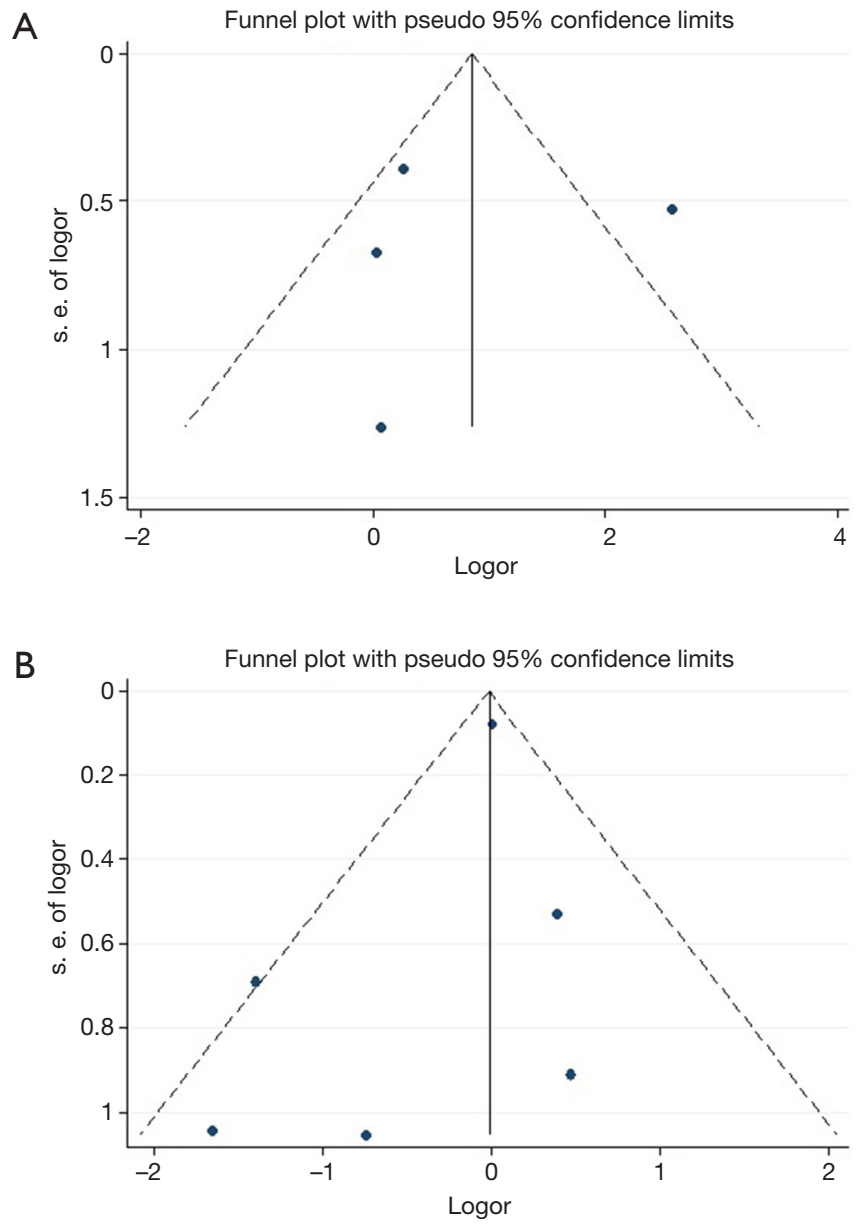

Figure S1 Funnel plot. (A) Funnel plot for publication bias assessment for mortality between LVAD destination therapy vs. heart transplantation; (B) Funnel plot for publication bias assessment for mortality between bridge to transplantation LVAD therapy vs. heart transplantation. 